\title{
Modelling the Transmission Dynamics of Severe Fever With Thrombocytopenia Syndrome in Jiangsu Province
}

\section{Nan Zhang}

Department of Acute Infectious Disease Control and Prevention, Jiangsu Provincial Centre for Disease Control and Prevention, Nanjing,Jiangsu, China

\section{Xiao-qing Cheng}

Department of Acute Infectious Disease Control and Prevention, Jiangsu Provincial Centre for Disease Control and Prevention, Nanjing, Jiangsu, China

\section{Bin Deng}

State Key Laboratory of Molecular Vaccinology and Molecular Diagnostics, School of Public Health, Xiamen University, Xiamen City 361102, Fujian Province, People's Republic of China

\section{Jia Rui}

State Key Laboratory of Molecular Vaccinology and Molecular Diagnostics, School of Public Health, Xiamen University, Xiamen City 361102, Fujian Province, People's Republic of China

\section{Luxia Qiu}

State Key Laboratory of Molecular Vaccinology and Molecular Diagnostics, School of Public Health, Xiamen University, Xiamen City 361102, Fujian Province, People's Republic of China

\section{Zeyu Zhao}

State Key Laboratory of Molecular Vaccinology and Molecular Diagnostics, School of Public Health, Xiamen University, Xiamen City 361102, Fujian Province, People's Republic of China

\section{Shengnan Lin}

State Key Laboratory of Molecular Vaccinology and Molecular Diagnostics, School of Public Health, Xiamen University, Xiamen City 361102, Fujian Province, People's Republic of China

\section{Xingchun Liu}

State Key Laboratory of Molecular Vaccinology and Molecular Diagnostics, School of Public Health, Xiamen University, Xiamen City 361102, Fujian province, People's Republic of China

\section{Jingwen Xu}

State Key Laboratory of Molecular Vaccinology and Molecular Diagnostics, School of Public Health, Xiamen University, Xiamen City 361102, Fujian Province, People's Republic of China

\section{Yao Wang}

State Key Laboratory of Molecular Vaccinology and Molecular Diagnostics, School of Public Health, Xiamen University, Xiamen City 361102, Fujian province, People's Republic of China

\section{Meng Yang}


State Key Laboratory of Molecular Vaccinology and Molecular Diagnostics, School of Public Health, Xiamen University, Xiamen City 361102, Fujian Province, People's Republic of China

\section{Yuanzhao Zhu}

: State Key Laboratory of Molecular Vaccinology and Molecular Diagnostics, School of Public Health, Xiamen University, Xiamen City 361102, Fujian Province, People's Republic of China

\section{Jiefeng Huang}

State Key Laboratory of Molecular Vaccinology and Molecular Diagnostics, School of Public Health, Xiamen University, Xiamen City 361102, Fujian Province, People's Republic of China

\section{Chan Liu}

State Key Laboratory of Molecular Vaccinology and Molecular Diagnostics, School of Public Health, Xiamen University, Xiamen City 361102, Fujian Province, People's Republic of China

\section{Weikang Liu}

State Key Laboratory of Molecular Vaccinology and Molecular Diagnostics, School of Public Health, Xiamen University, Xiamen City 361102, Fujian Province, People's Republic of China

\section{Li Luo}

State Key Laboratory of Molecular Vaccinology and Molecular Diagnostics, School of Public Health, Xiamen University, Xiamen City 361102, Fujian Province, People's Republic of China

\section{Zhuoyang Li}

State Key Laboratory of Molecular Vaccinology and Molecular Diagnostics, School of Public Health, Xiamen University, Xiamen City 361102, Fujian Province, People's Republic of China

\section{Peihua Li}

State Key Laboratory of Molecular Vaccinology and Molecular Diagnostics, School of Public Health, Xiamen University, Xiamen City 361102, Fujian Province, People's Republic of China

\section{Tianlong Yang}

State Key Laboratory of Molecular Vaccinology and Molecular Diagnostics, School of Public Health, Xiamen University, Xiamen City 361102, Fujian Province, People's Republic of China

\section{Zhi-feng Li}

Department of Acute Infectious Diseases Control and Prevention, Jiangsu Provincial Centre for Disease Control and Prevention, Nanjing, Jiangsu, China

\section{Shu-yi Liang}

Department of Acute Infectious Diseases Control and Prevention, Jiangsu Provincial Centre for Disease Control and Prevention, Nanjing, Jiangsu, China

\section{Xiao-chen Wang}

Department of Acute Infectious Diseases Control and Prevention, Jiangsu Provincial Centre for Disease Control and Prevention, Nanjing, Jiangsu, China

\section{Jian-li Hu}

Department of Acute Infectious Diseases Control and Prevention, Jiangsu Provincial Centre for Disease Control and Prevention, Nanjing, Jiangsu, China

\section{Tianmu Chen ( $\nabla 13698665 @ q q . c o m)$}


Xiamen University https://orcid.org/0000-0003-0710-5086

\section{Research}

Keywords: Severe fever with thrombocytopenia syndrome, Bunyavirus, SEIAR model of SFTS, Environment, Ticks

Posted Date: December 15th, 2020

DOl: https://doi.org/10.21203/rs.3.rs-127285/v1

License: (c) (1) This work is licensed under a Creative Commons Attribution 4.0 International License. Read Full License 
1 Modelling the transmission dynamics of Severe Fever with Thrombocytopenia

2 Syndrome in Jiangsu Province

3

4 Authors: Nan Zhang ${ }^{1 \#}$, Xiao-qing Cheng ${ }^{1 \#}$, Bin Deng ${ }^{2 \#}$, Jia Rui ${ }^{2 \#}$, Luxia Qiu ${ }^{2}$, Zeyu

$5 \mathrm{Zhao}^{2}$, Shengnan $\mathrm{Lin}^{2}$, Xingchun $\mathrm{Liu}^{2}$, Jingwen $\mathrm{Xu}^{2}$, Yao Wang ${ }^{2}$, Meng Yang ${ }^{2}$,

6 Yuanzhao $\mathrm{Zhu}^{2}$, Jiefeng Huang ${ }^{2}$, Chan $\mathrm{Liu}^{2}$, Weikang $\mathrm{Liu}^{2}, \mathrm{Li} \mathrm{Luo}^{2}$, Zhuoyang $\mathrm{Li}^{2}$,

7 Peihua $\mathrm{Li}^{2}$, Tianlong Yang ${ }^{2}$, Zhi-feng $\mathrm{Li}^{1}$, Shu-yi Liang ${ }^{1}$, Xiao-chen Wang ${ }^{1}$, Jian-li $\mathrm{Hu}^{1}$,

8 Tianmu Chen ${ }^{2 *}$

9

10

11

Affiliations:

${ }^{1}$ Department of Acute Infectious Diseases Control and Prevention, Jiangsu Provincial Centre for Disease Control and Prevention, Nanjing, Jiangsu, China

${ }^{2}$ State Key Laboratory of Molecular Vaccinology and Molecular Diagnostics, School of Public Health, Xiamen University, Xiamen City 361102, Fujian Province, People's Republic of China

${ }^{\#}$ N. Z., X. C., B. D., J. R. contributed equally to this work;

*Correspondence:

Tianmu Chen, State Key Laboratory of Molecular Vaccinology and Molecular Diagnostics, School of Public Health, Xiamen University, Xiamen City 361102, Fujian Province, People's Republic of China (13698665@qq.com).

Jian-li Hu, Jiangsu Provincial Centre for Disease Control and Prevention, 172, Jiangsu Rd, Nanjing 210009, Jiangsu Province, China (jshj1@jscdc.cn). 


\section{Abstract}

Background: Severe fever with thrombocytopenia syndrome (SFTS) is an emerging infectious disease that is regionally distributed in Asia with high fatality. Constructing the transmissibility model of SFTS could help provide clues for the intervention of disease control and fill the gap in the research of the SFTS model.

Methods: Based on the Susceptible-Exposed-Infectious-Symptomatic-Recovered (SEIAR) model and combined with the epidemiological characteristics of SFTS in Jiangsu Province. An SFTS transmission dynamics model was built to evaluate the effect through cutting off the different transmission routes and taking different interventions, to offer clues for disease prevention and control, and filled the gap in research of the SFTS model.

Results: For the transmission model of SFTS, the fitting effect of case data and model is great. Meanwhile, cutting off the transmission routes of the environment have the greatest effect on the prevention and control of SFTS, but isolation and shorten the course of the disease did not gain more effect.

Conclusions: This study is determined that cutting off the transmission routes from the environment to humans had the greatest effect on SFTS prevention and control.

\section{Keywords}

Severe fever with thrombocytopenia syndrome; Bunyavirus; SEIAR model of SFTS; Environment; Ticks 


\section{Introduction}

Severe fever with thrombocytopenia syndrome (SFTS) is an emerging infectious disease infected by a novel bunyavirus, which is called Severe Fever with Thrombocytopenia Syndrome Virus (SFTSV) [1]. The clinical manifestations of human infection have been characterized by high fever, a drastic reduction of platelets and leukocytes and hemorrhage[2, 3, 4, 5]. The clinical course is mainly divided into three stages: fever, multiple nerve dysfunction and recovery[1, 3, 4, 6, 7].

In addition, the circulating region of SFTS mainly covers the rural areas of central and northeast China, with a number of 500 1500 in 2011-2012 and a fatality rate of nearly $30 \%$. However, the fatality rate has dropped to $10 \% \sim 12 \%$ in recent years[8]. Besides, SFTS has also spread in South Korea and Japan. In South Korea, SFTS mainly occurred in tick-active months from May to September and infections reports continued until November, the fatality rate was above 35\% among 2012-2013 and the incidence rate dropped to $11.52 \%$ in 2016 [9]. In Japan, the cases were mainly reported in the western part, with a case fatality rate slightly higher than that in China (23\%) [10]. Currently, the SFTS fatality rate in Asia, seems to be decreasing but the incidence rate is increasing regionally, especially in China. According to the monitor data from China Information Network System of Disease Prevention and Control in 2010-2016, the incidence rate and prevalence of SFTS have increased year by year, SFTS cases have been found in 23 provinces in China[11]. Additionally, SFTS has not only brought the health burden, but also a certain amount of economic pressure. There was a study showing that patients and their families should pay nearly $\$ 3,158.4$ for their treatment, which is almost equal to the disposable income of rural residents in Anhui Province in 2017 [2]. Obviously, SFTS has become a significant threat to public health.

To fight against this emerging infectious disease, the domestic and foreign research of etiology and risk factors has made certain progress in recent years. According to the epidemiological characteristics of SFTS, some articles show that Henan Province, Hubei Province and Anhui Province are the mainly provinces, especially the border areas among these three provinces and the number of cases was largest[12]. The main infected population of SFTS is farmers who live in forested or hilly areas[13, 14, 15, 
16] and the main onset age is 50 years old and above. Recent research suggested that the Haemaphysalis longicornis[1, 17, 18, 19] is the most likely vector. The SFTS cases were distributed from March to November, but the peak period of disease focused on August and September. The seasonal distribution of SFTS cases was closely related to the seasonal trend of tick density[20, 21].

For the natural routes of transmission of SFTS, there is some preliminary research that comes up with the process of host animal-tick-human[22]. However, there was few systematic and definite research on transmission routes among vector, host and population of SFTSV. Meanwhile, various problems occurred in the treatment of SFTS such as unclear pathogenesis, no vaccine for prevention and no specific antiviral therapy for SFTS. As a result, we cannot keep the fatality rate at a low level. By far, there is no research on the transmission dynamics model of SFTS and no clear evidence for some preventive methods could achieve optimal intervention effect. Finding out the cause of the disease should be the first step, which means effective interventions and specific treatments should be positively researched to decrease the morbidity of SFTS, thus contributing to reducing the fatality rate.

This study aims to construct the Susceptible-Exposed-Infectious-AsymptomaticRecovered (SEIAR) model combination with the epidemiological characteristics of SFTS in Jiangsu Province and the transmission features of SFTS. In addition, the reported data of SFTS in Jiangsu Province from January 2001 to December 2019 was used to fit this model; the conditions were stimulated and the intervention effect of cutting different routes of transmission was assessed by setting the parameter of the model to obtain the best intervention plan, thus providing the clue to the intervention of disease control and filling the gap of the research of SFTS model.

\section{Method}

\section{Study design}

This study is divided into three sections. In section1, the transmission dynamics model of SFTS was established by searching scientific literature and analyzing data reported. In section 2, the data of reported cases was used to fit this model. In section 3, different conditions of cutting off transmission routes were stimulated and the effect of SFTS 
incidence was evaluated by simulating different interventions, see Figure 1.

\section{Establishment of transmission dynamics model}

According to the characteristics of epidemic and the route of transmission of SFTS in Jiangsu Province[23, 24, 25, 26], this study established SFTS transmissibility model in terms of days and based on the model of SEIAR. This model included four parts: human, tick, host animal and environment. Therefore, the human part is represented by subscript 1; The tick part is denoted by subscript 2; The host animal is represented by subscript 3; The environment is represented by subscript w, see Figure 2. The SEIAR model used in this study is based on the following assumptions:

\section{Human part of the SEIAR model}

1) This model supposes that disease belongs to vertical transmission and that the newborn of all kinds of people are all susceptible. And we suppose that the total population is $N$, the birth rate is $b r$, the natural mortality rate is $d r$. According to literature, the fatality rate of overt infections, $f$, is $16.1 \%[5]$. In the meanwhile, in the SEIAR model of SFTS, the mortality rate of all kinds of people, excluding overt infections, is low and the mortality rate of population SFTS is lower so we take the population-wide mortality rate to approximately replace the mortality rate of excluding overt infections. But the mortality rate of overt infections will be set as the sum of the mortality rate of the whole population and the mortality rate of SFTS.

2) According to literature, the means of SFTS transmission are limited to "human to human" transmission[27, 28], which can infect by contacting the blood[29] of patient or aerosol[30]. At the same time, the infectious ability of overt infection and latent infection can be different. So, we set the transmissibility of latent infection relative to overt infection as the $\kappa$ and the susceptible $S_{1}$ contact with two types of people as the incubation period $E_{1}$ that have some transmissibility and the infection rate coefficient is $\beta_{1}$.

3) We suppose that there are certain percentage the incubation period $(1-p) E_{1}(0 \leqq p \leqq$ 1) can transform to overt infections $I_{1}$ and at the same time other part of the incubation period $p E_{1}$ will become the latent infections $A_{1}$ after an incubation period. So, at time $t$, the development speed of the $E_{1}$ to $I_{1}$ pathway is proportional to the number of people 
134 in the incubation period, and the proportional coefficient is $(1-p) \omega_{1}$, the development speed of the $E_{1}$ to $A_{1}$ pathway is proportional to the number of people in the incubation period, and the proportional coefficient is $p \omega_{1}$.

4) The out speed of the population in the overt infections $I_{1}$ and latent infections $A_{1}$ are respectively proportional to their number of population and the ratio coefficients are respectively $\gamma$ and $\gamma^{\prime}$.

5) The infectors of SFTS will produce antibody after they are removed but this study supposes that this remover will never be infected and that set $R_{1}$ as the destination of the transmission route between people because the literature about the second infection in population was not found.

\section{Environment part of the SEIAR model}

1) There is no literature that can frankly prove that the environment is one of the transmission routes but there was evidence suggesting that in the environment, the possibility of infecting SFTS was higher than ticks[31, 32, 33, 34]. So, we assume that the environment has the ability to spread SFTS to humans and animals and we set it as $\beta_{w}[35]$.

2) As reported, the blood and excreta of human or host animals may diffuse the SFTS. And commonly SFTS have highly occurred in the wild, so we suggest that the animals have the possibility to infected the environment by their blood or excreta. We set the propagation coefficient $j$ as $10[36]$.

\section{Tick part of the SEIAR model}

1) According to literature, the SFTS as a natural focal disease, it mainly spread by ticks in nature and the Haemaphysalis longicornis Neumann is the dominant tick species[1, $17,18,19]$. The infectious ticks can spread the virus in the body by biting the host animal and can spread the disease in the population by directly biting the people[37].

2) As the Long-term storage host, ticks that infected SFTSV can lastingly preserve the pathogen and can keep the infectious ability of pathogen and have no symptoms[37]. So, this study set the transmission route among ticks as the Susceptible-ExposedAsymptomatic (SEA) model.

3) In the transmission among ticks, the infection rate coefficient is $\beta_{2}$. Our study 
considers that the SFTS was not diffused among ticks, so we set $\beta_{2}$ as 0 . The susceptible $S_{2}$ has some transmission ability that can make some infected ticks become the incubation period $E_{2}$ and the proportion of this part is $\beta_{2} S_{2} A_{2}$.

4) This study supposes that after one incubation period all the incubation period will transform to latent infection $A_{2}$, so, at time $t$, the number of the ticks that in the $E_{2}$ to $A_{2}$ route is $\omega_{2} E_{2}$.

5) The ticks that in the state of latent infection can spread the SFTSV further in the body of animals by biting them. Because the coefficient of infection rate that the virus spread from ticks to host animal is $\beta_{2-3}$ so the part of $\beta_{2-3} S_{3} A_{2}$ host animal can obtain the virus by tick's bite. In addition, those ticks can spread the SFTS among people by directly biting humans. Because the coefficient of infection rate that the virus spread from ticks to humans is $\beta_{2-1}$, so the part of $\beta_{2-1} S_{1} A_{2}$ people can save the virus by ticks directly bite.

\section{Host animal part of the SEIAR model}

1) According to the report, the main amplification hosts of arboviruses are small rodents which means after biting small rodents the arthropods can cause relative viremia; but the other uninfected arthropods can make the virus spread further by biting infected small rodents [38].

2) In SFTS, the ticks that infected SFTSV can cause viremia by biting host animals such as mice or domestic animals. Those uninfected ticks can be infected by biting infected host animal and the host animal has no symptoms after being infected with SFTSV[38, 39, 40, 41]. So, we still set the transmission progress of SFTSV as the SEA model in the part of host animal of transmission dynamics model.

3) Among the transmission of host animals, the infection rate coefficient is $\beta_{3}$. The susceptible $S_{3}$ has some infectious ability that can make some infected host animal transform to the incubation period $E_{3}$ and this ratio is $\beta_{3} S_{3} \mathrm{~A}_{3}$.

4) This study supposes that all the incubation period $E_{3}$ will transform to latent infection $A_{3}$ after one incubation period. At the time $t$, the number of host animals in the $E_{3}$ to $A_{3}$ pathway was $\omega_{3} E_{3}$.

5) The uninfected ticks can make the SFTSV spread further in the body of ticks after biting the host animal that is in the state of latent infection. The coefficient of host-to- 
194 tick transmission of the virus is $\beta_{3-2}$, so ticks with a $\beta_{3-2} S_{2} A_{3}$ component acquire the 195 virus by biting an infected host animal.

\section{The differential equation}

197 The above transmissibility model is expressed by differential equations as follows:

$198 \frac{d S_{1}}{d t}=b_{r} N_{1}-d_{r} S_{1}-\beta_{1} S_{1}\left(I_{1}+k A_{1}\right)-\beta_{21} S_{1} A_{2}-\beta_{w} S_{1} I_{w}-\beta_{31} S_{1} A_{3}$

$199 \frac{d E_{1}}{d t}=\beta_{1} S_{1}\left(I_{1}+k A_{1}\right)+\beta_{w} S_{1} I_{w}+\beta_{21} S_{1} A_{2}+\beta_{31} S_{1} A_{3}-d_{r} E_{1}-p \omega_{1} E_{1}$

$200-(1-p) \omega_{1} E_{1}$

$201 \quad \frac{d A_{1}}{d t}=p \omega_{1} E_{1}-d_{r} A_{1}-\gamma^{\prime} A_{1}$

$202 \frac{d I_{1}}{d t}=(1-p) \omega_{1} E_{1}-\gamma I_{1}-\left(d_{r}+f\right) I_{1}$

$203 \quad \frac{d R_{1}}{d t}=\gamma^{\prime} A_{1}+\gamma I_{1}-d_{r} R_{1}$

204

$205 \frac{d W}{d t}=j A_{3}$

206

$\frac{d S_{2}}{d t}=-\beta_{2} S_{2} A_{2}-\beta_{32} S_{2} A_{3}$

$208 \quad \frac{d E_{2}}{d t}=\beta_{2} S_{2} A_{2}+\beta_{32} S_{2} A_{3}-\omega_{2} E_{2}$

$209 \frac{d A_{2}}{d t}=\omega_{2} E_{2}$

210

$211 \frac{d S_{3}}{d t}=-\beta_{w} S_{3} W-\beta_{3} S_{3} A_{3}-\beta_{23} S_{3} A_{2}$

$212 \frac{d E_{3}}{d t}=\beta_{3} S_{3} A_{3}+\beta_{23} S_{3} A_{2}+\beta_{w} S_{3} W-\omega_{3} E_{3}$

$213 \frac{d A_{3}}{d t}=\omega_{3} E_{3}$

214 The left-hand side of the equation is respectively expressed as the instantaneous change

215 velocity of $S, E, I, A$ and $R$ at time $t$.

\section{Estimation of parameters}

217 The value of parameters: $p, 1 / \omega_{1}, 1 / \gamma, 1 / \gamma^{\prime}, f, 1 / \omega_{2}, 1 / \omega_{3}$ and $j$ all according to relative 218 literature. Among them, $p$ of recessive infection ranged from $3.2 \%$ to $5.5 \%[42]$, and the 219 mean was $4.3 \%$ in this study, i.e., $p=0.043$. And $1 / \omega_{1}$ of incubation period of SFTS in 
population ranged from 9 days to 14 days[43, 44], and the median was 11 days in this study. The course of the disease $1 / \gamma$ of overt infection was 2 weeks[45], i.e. 14 days. The research about the course of the disease $1 / \gamma^{\prime}$ of latent infection was not found, so using the same days with the course of the disease of overt infection, i.e., 14days. The fatality rate $\mathrm{f}$ ranged from $11.2 \%$ to $30 \%$, and $f=0.16[5]$. The incubation period after tick infection with SFTSV was $1 / \omega_{2}$ weeks, i.e., 7 days. The incubation period of SFTSV infection in host animals $1 / \omega_{3}$ was 12 days[40]. The coefficient of infection $\beta_{1}$ was generated by model fitting. No valid data or literature support was found for the relative transmissibility coefficient $\kappa$ of recessive infected persons. In this study, $\kappa=1$ was selected for calculation. The birth rate $(b r)$ and the death rate $(d r)$ were obtained from the statistical yearbook of Jiangsu Province, see Table 1.

\section{Evaluation of cutting off different transmission routes}

In this study, the transmissibility coefficient in the model was used to reflect different transmission routes, including transmissibility of environment to humans or host animals $\left(\beta_{w}\right)$, host animals to humans $\left(\beta_{3-1}\right)$, ticks to humans $\left(\beta_{2-1}\right)$, human to human $\left(\beta_{1}\right)$, ticks to host animals $\left(\beta_{2-3}\right)$, host animal to ticks $\left(\beta_{3-2}\right)$ and host animals to host animals $\left(\beta_{3}\right)$. Based on the established SFTS transmissibility model, using 2019 data, a scenario of cutting off various transmission routes is established by setting one of the above infection rates up to seven values to 0 at the time of simulation. We arranged the matrix between different transmission routes, as shown in Table 2, to perform a more comprehensive resection of transmission routes. The above methods are used to obtain the intervention effect of cutting off different transmission routes of SFTS, and the indicators of intervention effect include: cumulative number of cases, total attack rate, peak incidence site and peak incidence, to provide guidance for the prevention and control of the occurrence of SFTS epidemic.

\section{Evaluation of intervention effect}

In this study, the patients of SFTS took two interventions, isolation and shortened the disease course, and the model was established as the Figure 3. Among this, $Q$ was the number of people isolated, $R_{Q}$ was the recovery of isolation, was the isolation coefficient and the $c$ were the coefficient of shorting disease course in the model. We 
250 set the $\phi$ as the proportion of isolation which can be calculated by $\phi=\frac{R_{Q}}{R_{1}+R_{Q}}$; Then we 251 use $\gamma$ to represent shortened disease courses. In our study, we set $\phi$ as $0.5 \%, 5 \%$ and $25250 \%$; and set $\gamma$ as $1 / 14,1 / 12,1 / 10,1 / 8,1 / 6,1 / 4$. The two were combined in pairs to 253 judge the intervention effect of SFTS under different intervention intensity. And then set $\phi$ as $98 \%, 90 \%, 80 \%, 70 \%, 60 \%, 50 \%$ to simulate the solo effect of different isolation ratio. The indicators of intervention effect also included: cumulative number of cases, incidence rate, peak position of incidence and peak size of incidence. Finally, the optimal way to intervene in the spread of SFTS and provide guidance for the prevention and control of the outbreak of SFTS. Model simulation and statistical analysis

In this study, Excel was used for data summary and chart drawing, IBM SPSS Statistics (Version 21.0.0.0) was used for data statistical analysis, and Berkeley Madonna (Version 8.3.18) was used for fitting existing data and models, and epidemic prediction and evaluation were conducted for intervention effects under different transmission routes.

\section{Result}

\section{Model fitting}

The result of model fitting 2011-2019 SFTS incidence rate in Jiangsu Province was shown as Figure 4. We discovered that the incidence rate of SFTS has strictly seasonal changed by analyzed every month and years SFTS incidence rate, which means in a year the incidence rate of high in summer and autumn, often appear two peaks, but no cases in winter. Just as the result of model fitting, the SFTS incidence rate of 2011-2019 in Jiangsu Province was periodically changed. And it is often at rest in winter, begins to increase in spring, and peaks by July-August, and the peak incidence rate of SFTS in Jiangsu Province has increased to some extent in recent years compared with 20112014. The $R^{2}$ and $P$ values of the model fitting results for $2011-2019$ were listed in Table 3, and it can see that the model fitting results for all years were good and $P$ was significant. $(P<0.05)$.

\section{Model simulation}




\section{Cutting transmission routes}

The simulation results of cutting off different transmission routes based on the 2019 SFTS transmission dynamics model in Jiangsu Province are shown in Table 4. When the route of transmission between people is cut off, SFTS cannot spread in the population, so other combinations containing $\beta_{1}$ are not shown. It can see that when the transmissibility of environment $(\beta w)$ is cut off, the transmissibility of SFTS in the population is very weak, so its four evaluation indicators are low. In cutting off multiple transmission routes, it was found that the transmission of SFTS in the human population was lower when the transmission of the environment and animals was cut off at the same time. And as more transmission routes are cut off, SFTS becomes less transmissible in the population, see Table 4.

\section{Taking different interventions}

It can be seen that both interventions, taking isolation and shortening the course of the disease, have not limited the spread of SFTS regardless of the combination of interventions. And no matter how many isolation ratios we applied, taken isolation also had little effect on the number of SFTS cases. There will only be a case reduction if the isolation is combined to shorten the course of disease, but the cumulative number of cases has fallen by only one. There was no difference in the incidence rate of SFTS between different degrees of isolation or shortening the course of the disease, see Table 5,6 .

\section{Discussion}

SFTS is an emerging infectious disease caused by a novel bunyavirus, which was termed severe fever with thrombocytopenia syndrome virus (SFTSV). Since it was first discovered in China in 2009, the incidence rate has been increasing year by year, and the coverage of the epidemic area has expanded. Data from the Chinese CDC information system from 2010 to 2016 showed that Jiangsu Province is one of the provinces with the highest incidence of SFTS. Since the first confirmed case of SFTS discovered in 2010, Jiangsu Province has conducted stringenter SFTS monitoring. Therefore, based on the SFTS monitoring data of Jiangsu Province from 2011 to 2019, the SFTS transmission dynamics model was established to use the model to simulate 
the change of incidence rate by cutting different transmission routes and taking interventions. We assessed the effect of those interventions and its effects are of great significance for preventing and controlling the occurrence of SFTS.

\section{The effect of cutting different transmission routes}

Previous studies have shown that the number of SFTS patients infected by the environment were more than those who were infected by ticks[17, 31, 32, 33, 34]. But most studies only use the density of ticks in the environment and the anti-body of SFTSV in ticks to evaluate the hazardous situation of environment $[46,47,48,49]$, so they do not consider the environment as one of the transmission routes. As we all know, several factors such as the number of ticks, the dominant phase of ticks, outdoor activities and the change of weather can affect the occurrence of SFTS[50]. There was one study collected SFTSV from the inanimate things in the room of SFTS patients, which can consider the environment could be one of the transmission routes of SFTS, although the test result of the air sample was negative[51]. Therefore, we established a SFTS transmissibility model and used the coefficient of transmissibility to reflect the different ways of transmission. Based on the literature and the data of SFTS incidents in Jiangsu province, 2019. We simulated cutting different transmission ways to show the different effects of interventions. The result of this study showed that in all the combination of cutting different transmission routes, the cumulative number of SFTS cases and other indicators could be significantly reduced when the transmission route containing the environment was cutoff. Moreover, the intervention effect is more obvious when the combination of environmental and host animal transmission routes is cut simultaneously. Those results indicated that the environment is an important route for the transmission of SFTS, this was consistent with previous research. Besides, this also provides a direction that can be explained. The reason may be that people were not exposed to wild environment, and the chance of contacting ticks or animal excreta, blood, etc., decreases, which leads to the low incidence rate of SFTS. In addition, some literature has shown that SFTS was an environment-tick-host animal-tick cycle and that humans were infected by accidental participation in one of the progress. Therefore, the more transmission ways we cut off, the smaller the impact of SFTS was. Interestingly, 
the effect of cutting off the environment and the transmission route of ticks on SFTS was less than that of cutting off the environment and host animals simultaneously. This might be the main way for people to be infected with SFTS except for tick bites. In previous studies, there was also literature showing that those infected with SFTS with a clear history of tick bites only accounted for a small proportion (about 22\%-29\%[6, $34,52])$ of all infected individuals. The study also suggested that humans might be infected with SFTS after exposure to animal blood[6]. Although the transmission of SFTS was initiated by ticks, it may be that the two transmission routes of animal and environment are more important for human beings.

\section{The effect of taking interventions}

For infectious diseases, there are three links and two factors. Three links include the source of infection, transmission routes, the population; two factors include natural factors and social factors. Common measures to control infectious diseases include controlling the source of infection, cutting off the route of transmission and protecting the population. Therefore, after cutting off the route of transmission and protecting the population, we also need to control the source of infection, and here we choose to take interventions including taking isolation and shortening the course of the disease. Besides, we added the effects of taking isolation and shortening the course of the disease according to the established SFTS transmissibility model. Based on the data of SFTS in 2019 , the results of the simulation were used to evaluate the effect of the two interventions on SFTS. The results showed that whether taking isolation alone or shortening the course of the disease, or combining the two interventions, the effect of interventions mentioned above on the incidence of SFTS was limited. And the different isolation ratio has little effect on the cases. The reason for this may be that the incidence of SFTS is low, and most of the patients are farmers, or field workers[17, 33, 34, 50]. For people living in cities, they are less likely to be infected with SFTSV. And even human-to-human transmission, the greatest chance of occurrence was only the population who contact its blood, such as nurses or family members[53]. In addition, secondary SFTS infection leads to milder clinical manifestations compared with primary SFTS. Therefore, secondary patients only experience sudden fever, 
thrombocytopenia, leukopenia, and gastrointestinal symptoms and recover more quickly[27].

In summary, taking isolation and shortening the course of the disease have little impact on SFTS, which may suggest that prevention and control of the development of SFTS should focus on cutting off the direction of transmission routes. Therefore, according to the results of cutting off the transmission route earlier, the possibility of traveling to the wild environment should first be avoided in order to prevent and control the transmission of SFTS. For the population living in rural areas or taking exercise in the wild, self-protection should be done well, such as wearing protective clothing and regularly cleaning the animal feces around the residence. What's more, in order to reduce the possibility of infection from domestic animals, livestock should be placed in certain places as far as possible to prevent its contact with the field environment.

\section{Limitation}

There are some limitations in our study. First, the data of SFTS cases collected by the National Disease Surveillance Information Reporting Management System, which the capability and availability of this system is finite. Second, this study failed to separate the effects of ticks and host animals on humans in the environment. Third, some perimeters of our model are collected from literature that may be a certain error in its timeliness.

\section{Conclusion}

In this study, the transmission dynamics model of SFTS was constructed based on the epidemiological characteristics of SFTS in Jiangsu Province, the effect of cutting off different transmission routes and taking isolation and shortening course of disease on SFTS control was evaluated. Finally, it was determined that cutting off the transmission route from environment to human had the greatest effect on SFTS prevention and control. 
SFTS: Severe fever with thrombocytopenia syndrome; SFTSV: Severe Fever with Thrombocytopenia Syndrome Virus; SEIAR: Susceptible-Exposed-InfectiousAsymptomatic-Recovered; SEA: Susceptible-Exposed-Asymptomatic; CDC: Center for Disease Control and Prevention;

\section{Acknowledgements}

We thank the staff members at the hospitals, local health departments, and municipal and county-level Center for Disease Control and Prevention offices for their valuable assistance in coordinating the data collection.

Ethics approval and consent to participate

This effort of disease control was part of CDC's routine responsibility in Jiangsu Province, China. Therefore, institutional review and informed consent were not

\section{Consent for publication}

Not applicable.

\section{Availability of data and material}

Data supporting the conclusions of this article are included within the article.

\section{Competing interests}

The authors declare that they have no competing interests.

\section{Funding}

This work was partly supported by the Bill \& Melinda Gates Foundation (INV-005834), the Science and Technology Program of Fujian Province (No: 2020Y0002) and the Xiamen New Coronavirus Prevention and Control Emergency Tackling Special Topic 
No.81601794; ZF Li: No.81703284), Jiangsu Provincial Nature Science Foundation (ZF Li: No. BK20161584) and "Six One Project” Top Talent Research Plan of Jiangsu High Level Health Talents (JL Hu: LGY2019073)

\section{Authors' contributions}

TMC, JR and BD designed research; NZ, JR, BD, CL, JFH, ZYL, WKL, LL, PHL, TLY and XQC analyzed data, TMC, JR, BD, LXQ, ZYZ, SNL, MY, YZZ, YW, JWX, JFH, ZYL, ZFL, SYL and JLH conducted the research and analyzed the results; TMC, JLH, NZ, JR and BD wrote the manuscript. All authors read and approved the final manuscript.

\section{Reference}

1. Yu XJ, Liang MF, Zhang SY, Liu Y, Li JD, Sun YL, et al. Fever with thrombocytopenia associated with a novel bunyavirus in China. N Engl J Med. 2011;364 16:1523-32; doi: 10.1056/NEJMoa1010095. https://www.ncbi.nlm.nih.gov/pubmed/21410387.

2. Gong L, Wang J, Zhang Y, Zhang L, Lyu Y, Ma W, et al. Socioeconomic burden of severe fever with thrombocytopenia syndrome in endemic areas of Anhui Province, eastern China. Zoonoses Public Health. 2019;66 7:879-85; doi: 10.1111/zph.12634. https://www.ncbi.nlm.nih.gov/pubmed/31334609.

3. Zhan JB, Cheng J, Hu B, Li J, Pan RG, Yang ZH, et al. Pathogens and epidemiologic feature of severe fever with thrombocytopenia syndrome in Hubei province, China. Virus Res. 2017;232:63-8; doi: 10.1016/j.virusres.2017.01.009. < Go to ISI>://WOS:000400532700009.

4. Li H, Lu QB, Xing B, Zhang SF, Liu K, Du J, et al. Epidemiological and clinical features of laboratory-diagnosed severe fever with thrombocytopenia syndrome in China, 2011-17: a prospective observational study. Lancet Infect Dis. 2018;18 10:1127-37; doi: 10.1016/S1473-3099(18)30293-7. <Go to ISI $>: / / W O S: 000445774200035$.

5. Wang L, Wan G, Shen Y, Zhao ZH, Lin L, Zhang W, et al. A nomogram to predict mortality in patients with severe fever with thrombocytopenia syndrome at the early stage-A multicenter study in China. Plos Neglect Trop D. 2019;13 11; doi: ARTN e000782910.1371/journal.pntd.0007829. $<$ Go to ISI $>$ ://WOS:000503278100039.

6. Li DX. Severe fever with thrombocytopenia syndrome: a newly discovered emerging infectious disease. Clin Microbiol Infec. 2015;21 7:614-20; doi: 10.1016/j.cmi.2015.03.001. $<$ Go to ISI $>$ ://WOS:000357752700002.

7. Li ZF, Hu JL, Cui LBA, Hong Y, Liu JW, Li PF, et al. Increased Prevalence of Severe Fever with Thrombocytopenia Syndrome in Eastern China Clustered with Multiple Genotypes and Reasserted Virus during 2010-2015. Sci Rep-Uk. 
2017;7; doi: ARTN 650310.1038/s41598-017-06853-1. <Go to ISI>://WOS:000406364600016.

8. Silvas JA, Aguilar PV. The Emergence of Severe Fever with Thrombocytopenia Syndrome Virus. Am J Trop Med Hyg. 2017;97 4:992-6; doi: 10.4269/ajtmh.160967. https://www.ncbi.nlm.nih.gov/pubmed/28820686.

9. Im JH, Baek J, Durey A, Kwon HY, Chung MH, Lee JS. Current Status of TickBorne Diseases in South Korea. Vector Borne Zoonotic Dis. 2019;19 4:225-33; doi: 10.1089/vbz.2018.2298. https://www.ncbi.nlm.nih.gov/pubmed/30328790.

10. Kimura T, Fukuma A, Shimojima M, Yamashita Y, Mizota F, Yamashita M, et al. Seroprevalence of severe fever with thrombocytopenia syndrome (SFTS) virus antibodies in humans and animals in Ehime prefecture, Japan, an endemic region of SFTS. J Infect Chemother. 2018;24 10:802-6; doi: 10.1016/j.jiac.2018.06.007. https://www.ncbi.nlm.nih.gov/pubmed/30017796.

11. Zhan JB, Wang Q, Cheng J, Hu B, Li J, Zhan FX, et al. Current status of severe fever with thrombocytopenia syndrome in China. Virol Sin. 2017;32 1:51-62; doi: 10.1007/s12250-016-3931-1. <Go to ISI >://WOS:000396155400007.

12. Gai ZT, Liang MG, Zhang Y, Zhang S, Jin C, Wang SW, et al. Person-to-Person Transmission of Severe Fever With Thrombocytopenia Syndrome Bunyavirus Through Blood Contact. Clin Infect Dis. 2012;54 2:249-52; doi: 10.1093/cid/cir776. $<$ Go to ISI $>$ ://WOS:000298383700015.

13. YUAN Chun CN, WANG Bing-jun, LI Wei, ZHANG Lan. Clinical analysis of 253 patients with severe fever with thrombocytopenia syndrome. China Journal of Modern Medicine. 2011:3413-7; doi: CNKI:SUN:ZXDY.0.2011-27-023.

14. Ding Shu-jun LY, Zhang Xiao-mei, Wang Xian-jun, Yu Xue-jie. Research advances on epidemiology of severe fever with thrombocytopenia syndrome. Chinese Journal of Zoonoses. 2014; doi: 10.3969/cjz.j.issn.

15. Liu W, Lu QB, Cui N, Li H, Wang LY, Liu K, et al. Case-Fatality Ratio and Effectiveness of Ribavirin Therapy Among Hospitalized Patients in China Who Had Severe Fever With Thrombocytopenia Syndrome. Clin Infect Dis. 2013;57 9:1292-9; doi: 10.1093/cid/cit530. < Go to ISI >://WOS:000325762500016.

16. You Ai-guo YJ-h, Du Yan-hua, Wang Hai-feng, Tang Xiao-yan, Chen Hao-min, $\mathrm{Xu}$ Bian-li. Research advances on epidemiology of severe fever with thrombocytopenia syndromeAnalysis of the epidemiological and pathogenic characteristics of severe fever with thrombocytopenia syndrome (SFTS) in Henan Province of China, 2012-2013. Journal of pathogen Biology. 2014:5525; doi: Journal of pathogen Biology.

17. Li DX. A highly pathogenic new bunyavirus emerged in China. Emerg Microbes Infec. 2013;2; doi: ARTN e110.1038/emi.2013.1. <Go to ISI>://WOS:000339177300001.

18. Zhang YZ, Zhou DJ, Xiong Y, Chen XP, He YW, Sun Q, et al. Hemorrhagic fever caused by a novel tick-borne Bunyavirus in Huaiyangshan, China. Zhonghua Liu Xing Bing Xue Za Zhi. 2011;32 3:209-20. https://www.ncbi.nlm.nih.gov/pubmed/21457654.

19. Zhang YZ, Zhou DJ, Qin XC, Tian JH, Xiong YW, Wang JB, et al. The Ecology, 
Genetic Diversity, and Phylogeny of Huaiyangshan Virus in China. J Virol. 2012;86 5:2864-8; doi: 10.1128/Jvi.06192-11. <Go to ISI>://WOS:000300536800045.

20. Klompen JSH, Black WC, Keirans JE, Oliver JH. Evolution of ticks. Annu Rev Entomol. 1996;41:141-61; doi: DOI 10.1146/annurev.en.41.010196.001041. $<$ Go to ISI $>$ ://WOS:A1996TP40300008.

21. LIU Y HXY, DU Y H. Survey on ticks and detection of new bunyavirus in some vect in the endemic areas of fever, thrombocytopenia and leukopenia syndrome (FTLS) in Henan province. Chinese journal of preventive medicine. 2012:5004.

22. Yamaji K, Aonuma H, Kanuka H. Distribution of tick-borne diseases in Japan: Past patterns and implications for the future. J Infect Chemother. 2018;24 7:499504; doi:

10.1016/j.jiac.2018.03.012. https://www.ncbi.nlm.nih.gov/pubmed/29685854.

23. Hu J, Li Z, Cai J, Liu D, Zhang X, Jiang R, et al. A Cluster of BunyavirusAssociated Severe Fever With Thrombocytopenia Syndrome Cases in a Coastal Plain Area in China, 2015: Identification of a Previously Unidentified Endemic Region for Severe Fever With Thrombocytopenia Bunyavirus. Open Forum Infect Dis. 2019;6 6:ofz209; doi: 10.1093/ofid/ofz209. https://www.ncbi.nlm.nih.gov/pubmed/31211156.

24. Li Z, Hu J, Bao C, Li P, Qi X, Qin Y, et al. Seroprevalence of antibodies against SFTS virus infection in farmers and animals, Jiangsu, China. J Clin Virol. 2014;60 3:185-9; $\quad$ doi: $\quad 10.1016 /$ j.jcv.2014.03.020. https://www.ncbi.nlm.nih.gov/pubmed/24793967.

25. Li Z, Hu J, Cui L, Hong Y, Liu J, Li P, et al. Increased Prevalence of Severe Fever with Thrombocytopenia Syndrome in Eastern China Clustered with Multiple Genotypes and Reasserted Virus during 2010-2015. Sci Rep. 2017;7 1:6503; doi: 10.1038/s41598-017-06853-1. https://www.ncbi.nlm.nih.gov/pubmed/28747674.

26. Zhang D, Sun C, Yu H, Li J, Liu W, Li Z, et al. Environmental Risk Factors and Geographic Distribution of Severe Fever with Thrombocytopenia Syndrome in Jiangsu Province, China. Vector Borne Zoonotic Dis. 2019;19 10:758-66; doi: 10.1089/vbz.2018.2425. https://www.ncbi.nlm.nih.gov/pubmed/30994412.

27. Bao CJ, Qi X, Wang H. A novel bunyavirus in China. N Engl J Med. 2011;365 9:862-3; author reply 4-5; doi: 10.1056/NEJMc1106000. https://www.ncbi.nlm.nih.gov/pubmed/21879913.

28. Chen $\mathrm{HB}, \mathrm{Hu} \mathrm{K}$, Zou JJ, Xiao JX. A cluster of cases of human-to-human transmission caused by severe fever with thrombocytopenia syndrome bunyavirus. International Journal of Infectious Diseases. 2013;17 3:E206-E8; doi: 10.1016/j.ijid.2012.11.006. < Go to ISI>://WOS:000315205000013.

29. Wang YL, Deng BC, Zhang J, Cui W, Yao WQ, Liu P. Person-to-Person Asymptomatic Infection of Severe Fever with Thrombocytopenia Syndrome Virus through Blood Contact. Internal Med. 2014;53 8:903-6; doi: 10.2169/internalmedicine.53.1164. $<$ Go to ISI>://WOS:000334674400017. 
30. Gong Z, Gu S, Zhang Y, Sun J, Wu X, Ling F, et al. Probable aerosol transmission of severe fever with thrombocytopenia syndrome virus in southeastern China. Clin Microbiol Infec. 2015;21 12:1115-20; doi: 10.1016/j.cmi.2015.07.024. <Go to ISI>://WOS:000366585900023.

31. Ding F, Guan XH, Kang K, Ding SJ, Huang LY, Xing XS, et al. Risk Factors for Bunyavirus-Associated Severe Fever with Thrombocytopenia Syndrome, China. Plos Neglect Trop D. 2014;8 10; doi: ARTN e326710.1371/journal.pntd.0003267. $<$ Go to ISI $>$ ://WOS:000344589000071.

32. Ding F, Zhang WY, Wang LY, Hu WB, Magalhaes RJS, Sun HL, et al. Epidemiologic Features of Severe Fever With Thrombocytopenia Syndrome in China, 2011-2012. Clin Infect Dis. 2013;56 11:1682-3; doi: 10.1093/cid/cit100. $<$ Go to ISI $>$ ://WOS:000318641300031.

33. Liu K, Zhou H, Sun RX, Yao HW, Li Y, Wang LP, et al. A National Assessment of the Epidemiology of Severe Fever with Thrombocytopenia Syndrome, China. Sci Rep-Uk. 2015;5; doi: ARTN 967910.1038/srep09679. <Go to ISI $>$ ://WOS:000353333000001.

34. Sun JM, Chai CL, Lv HK, Lin JF, Wang CW, Chen EF, et al. Epidemiological characteristics of severe fever with thrombocytopenia syndrome in Zhejiang Province, China. International Journal of Infectious Diseases. 2014;25:180-5; doi: 10.1016/j.ijid.2014.02.022. < Go to ISI >://WOS:000343815700039.

35. Hui MX-rM. Epidemiological Characteristics of Febrile Thrombocytopenia Syndrome. Chinese Journal of Thrombosis and Hemostasis. 2019:268-9; doi: 10.3969/j.issn.1009-6213.2019.02.033.

36. Jiang X-1: An investigation of viral vectors and hosts in "Fever with Thrombocytopenia syndrome". Shandong university,; 2012.

37. Yun SM, Lee WG, Ryou J, Yang SC, Park SW, Roh JY, et al. Severe Fever with Thrombocytopenia Syndrome Virus in Ticks Collected from Humans, South Korea, 2013. Emerg Infect Dis. 2014;20 8:1358-61; doi: 10.3201/eid2008.131857. < Go to ISI $>$ ://WOS:000339864000017.

38. Li ZF, Hu JL, Bao CJ, Li PF, Qi X, Qin YF, et al. Seroprevalence of antibodies against SFTS virus infection in farmers and animals, Jiangsu, China. J Clin Virol. 2014;60 3:185-9; doi: 10.1016/j.jcv.2014.03.020. $<$ Go to ISI $>: / / W O S: 000339287200001$.

39. Ni HX, Yang F, Li YD, Liu WB, Jiao SL, Li ZX, et al. Apodemus agrarius is a potential natural host of severe fever with thrombocytopenia syndrome (SFTS)causing novel bunyavirus. J Clin Virol. 2015;71:82-8; doi: 10.1016/j.jcv.2015.08.006. $<$ Go to ISI $>$ ://WOS:000361145400015.

40. Niu GY, Li JD, Liang MF, Jiang XL, Jiang M, Yin HY, et al. Severe Fever with Thrombocytopenia Syndrome Virus among Domesticated Animals, China. Emerg Infect Dis. 2013;19 5:756-63; doi: 10.3201/eid1905.120245. $<$ Go to ISI $>$ ://WOS:000328173400008.

41. Diang SJ, Yin HY, Xu XH, Liu GS, Jiang SX, Wang WQ, et al. A CrossSectional Survey of Severe Fever with Thrombocytopenia Syndrome Virus Infection of Domestic Animals in Laizhou City, Shandong Province, China. Jpn 
J Infect Dis. 2014;67 1:1-4; doi: DOI 10.7883/yoken.67.1. <Go to ISI>://WOS:000330751500001.

42. Li P, Tong ZD, Li KF, Tang A, Dai YX, Yan JB. Seroprevalence of severe fever with thrombocytopenia syndrome virus in China: A systematic review and metaanalysis. Plos One. 2017;12 4; doi: ARTN e017559210.1371/journal.pone.0175592. $<$ Go ISI>://WOS:000399949000052.

43. Gu S, Wu X, Zhou B, Ling F, Zhang H, Huang Y, et al. [Epidemiological investigation on an outbreak of severe fever with thrombocytopenia syndrome in northwest Zhejiang province]. Zhonghua Liu Xing Bing Xue Za Zhi. 2015;36 4:364-7. https://www.ncbi.nlm.nih.gov/pubmed/25975551.

44. Kurihara S. [Severe Fever with Thrombocytopenia Syndrome]. Rinsho Byori. 2016;64 9:1052-6. https://www.ncbi.nlm.nih.gov/pubmed/30609458.

45. Liu S, Chai C, Wang C, Amer S, Lv H, He H, et al. Systematic review of severe fever with thrombocytopenia syndrome: virology, epidemiology, and clinical characteristics. Rev Med Virol. 2014;24 2:90-102; doi: 10.1002/rmv.1776. https://www.ncbi.nlm.nih.gov/pubmed/24310908.

46. Ham H, Jo S, Jang J, Choi S. No Detection of Severe Fever with Thrombocytopenia Syndrome Virus from Ixodid Ticks Collected in Seoul. Korean J Parasitol. 2014;52 2:221-4; doi: 10.3347/kjp.2014.52.2.221. <Go to ISI>://WOS:000335278500017.

47. Kim BJ, Kim H, Won S, Kim HC, Chong ST, Klein TA, et al. Ticks Collected from Wild and Domestic Animals and Natural Habitats in the Republic of Korea. Korean J Parasitol. 2014;52 3:281-5; doi: 10.3347/kjp.2014.52.3.281. $<$ Go to ISI>://WOS:000338751500007.

48. Kim-Jeon MD, Jegal S, Jun H, Jung H, Park SH, Ahn SK, et al. Four Year Surveillance of the Vector Hard Ticks for SFTS, Ganghwa-do, Republic of Korea. Korean J Parasitol. 2019;57 6:691-8; doi: 10.3347/kjp.2019.57.6.691. $<$ Go to ISI $>$ ://WOS:000508352900019.

49. Nakayama S, Kobayashi T, Nakamura A, Yoshitomi H, Song YDJ, Ashizuka Y. Detection of Borrelia DNA in Tick Species Collected from Vegetation and Wild Animals in Fukuoka, Japan. Jpn J Infect Dis. 2020;73 1:61-4; doi: 10.7883/yoken.JJID.2019.146. <Go to ISI >://WOS:000514021800011.

50. Li ZF, Bao CJ, Hu JL, Liu WD, Wang XC, Zhang L, et al. Ecology of the TickBorne Phlebovirus Causing Severe Fever with Thrombocytopenia Syndrome in an Endemic Area of China. Plos Neglect Trop D. 2016;10 4; doi: ARTN e000457410.1371/journal.pntd.0004574. $<$ Go to ISI>://WOS:000375376700024.

51. Ryu BH, Kim JY, Kim T, Kim MC, Kim MJ, Chong YP, et al. Extensive severe fever with thrombocytopenia syndrome virus contamination in surrounding environment in patient rooms. Clin Microbiol Infec. 2018;24 8; doi: ARTN 911.e110.1016/j.cmi.2018.01.005. <Go to ISI>://WOS:000439403900025.

52. Xing XS, Guan XH, Liu L, Xu JQ, Li GM, Zhan JB, et al. A Case-control Study of Risk Sources for Severe Fever with Thrombocytopenia Syndrome in Hubei 
Province, China. International Journal of Infectious Diseases. 2017;55:86-91; doi: 10.1016/j.ijid.2017.01.003. <Go to ISI >://WOS:000397212000016.

53. Jia B, Wu WH, Huang R, Wang GY, Song PX, Li Y, et al. Characterization of clinical features and outcome for human-to-human transmitted severe fever with thrombocytopenia syndrome. Infect Dis-Nor. 2018;50 8:601-8; doi: 10.1080/23744235.2018.1449962. < Go to ISI >://WOS:000438401700004. 
649 Figure legends

650 Figure 1 Study design map

651 Figure 2 SFTS without intervention model

652 Figure 3 SFTS with intervention model

653 Figure 4 Model fitting result of incidence of 2011-2019 in Jiangsu Province 654 
655 Tables

656 Table 1 Definition and value of parameters

\begin{tabular}{|c|c|c|c|c|c|}
\hline & $\begin{array}{l}\text { para } \\
\text { mete } \\
\mathrm{r}\end{array}$ & definition & value & unite & method \\
\hline \multirow{9}{*}{ Person } & $\beta_{1}$ & $\begin{array}{l}\text { Person-to- } \\
\text { person } \\
\text { transmissibility } \\
\text { coefficient }\end{array}$ & - & $\left(\right.$ person*day) ${ }^{-1}$ & model fitting \\
\hline & $\kappa$ & $\begin{array}{l}\text { Relative } \\
\text { transmissibility } \\
\text { coefficient of } \\
\text { inapparent } \\
\text { infection }\end{array}$ & 1 & 1 & - \\
\hline & $\mathrm{p}$ & $\begin{array}{l}\text { Proportion of } \\
\text { latent infection }\end{array}$ & 0.043 & 1 & Ref.[42] \\
\hline & $1 / \omega_{1}$ & $\begin{array}{l}\text { Incubation } \\
\text { period }\end{array}$ & 11 & day & Ref.[43] \\
\hline & $1 / \gamma$ & $\begin{array}{l}\text { Infectious } \\
\text { period of } \\
\text { dominant } \\
\text { infection }\end{array}$ & 14 & day & Ref.[45] \\
\hline & $1 / \gamma^{\prime}$ & $\begin{array}{l}\text { Latent infection } \\
\text { period }\end{array}$ & 14 & day & - \\
\hline & $f$ & Fatality rate & 0.16 & 1 & Ref.[5] \\
\hline & $b r$ & Birth rate & $\begin{array}{l}0.0094 \\
81\end{array}$ & 1 & $\begin{array}{l}\text { statistical Yearbook } \\
\text { of Jiangsu Province }\end{array}$ \\
\hline & $d r$ & Mortality rate & $\begin{array}{l}0.0070 \\
03\end{array}$ & 1 & $\begin{array}{l}\text { statistical Yearbook } \\
\text { of Jiangsu Province }\end{array}$ \\
\hline
\end{tabular}




\begin{tabular}{|c|c|c|c|c|c|}
\hline \multirow{4}{*}{ Ticks } & $\beta_{2}$ & $\begin{array}{l}\text { Coefficient of } \\
\text { transmissibility } \\
\text { between ticks }\end{array}$ & 0 & $\left(\text { pieces }^{*} \text { day }\right)^{-1}$ & - \\
\hline & $\beta_{2-1}$ & $\begin{array}{l}\text { Coefficient of } \\
\text { tick-to-human } \\
\text { transmissibility }\end{array}$ & $8 \beta_{1}$ & $\left(\text { person}^{*} \text { day }\right)^{-1}$ & Ref. [35] \\
\hline & $\beta_{2-3}$ & $\begin{array}{l}\text { Coefficient of } \\
\text { tick-to-host } \\
\text { infection }\end{array}$ & $8 \beta_{1}$ & $\left(\text { pieces }^{*} \text { day }\right)^{-1}$ & Ref.[35] \\
\hline & $1 / \omega_{2}$ & $\begin{array}{l}\text { Incubation } \\
\text { period of ticks }\end{array}$ & 7 & day & Ref. [26] \\
\hline \multirow{5}{*}{$\begin{array}{l}\text { Host } \\
\text { animal }\end{array}$} & $\beta_{3}$ & $\begin{array}{l}\text { Coefficient of } \\
\text { transmissibility } \\
\text { between host } \\
\text { animal }\end{array}$ & $2 \beta_{1}$ & $\left(\text { pieces }^{*} \text { day }\right)^{-1}$ & Ref.[35] \\
\hline & $\beta_{3-1}$ & $\begin{array}{l}\text { Coefficient of } \\
\text { transmissibility } \\
\text { rate of host } \\
\text { animal to } \\
\text { human }\end{array}$ & $2 \beta_{1}$ & $\left(\text { pieces }^{*} \text { day }\right)^{-1}$ & Ref.[35] \\
\hline & $\beta_{3-2}$ & $\begin{array}{l}\text { Coefficient of } \\
\text { host animal } \\
\text { infection to } \\
\text { ticks }\end{array}$ & $8 \beta_{1}$ & $(\text { person*day })^{-1}$ & Ref.[35] \\
\hline & $1 / \omega_{3}$ & $\begin{array}{l}\text { Host animal } \\
\text { incubation } \\
\text { period }\end{array}$ & 12 & day & Ref.[40] \\
\hline & $j$ & $\begin{array}{l}\text { Rate of host } \\
\text { animal }\end{array}$ & 10 & - & Ref.[36] \\
\hline
\end{tabular}


discharge to the

environment

Coefficient of

transmissibility

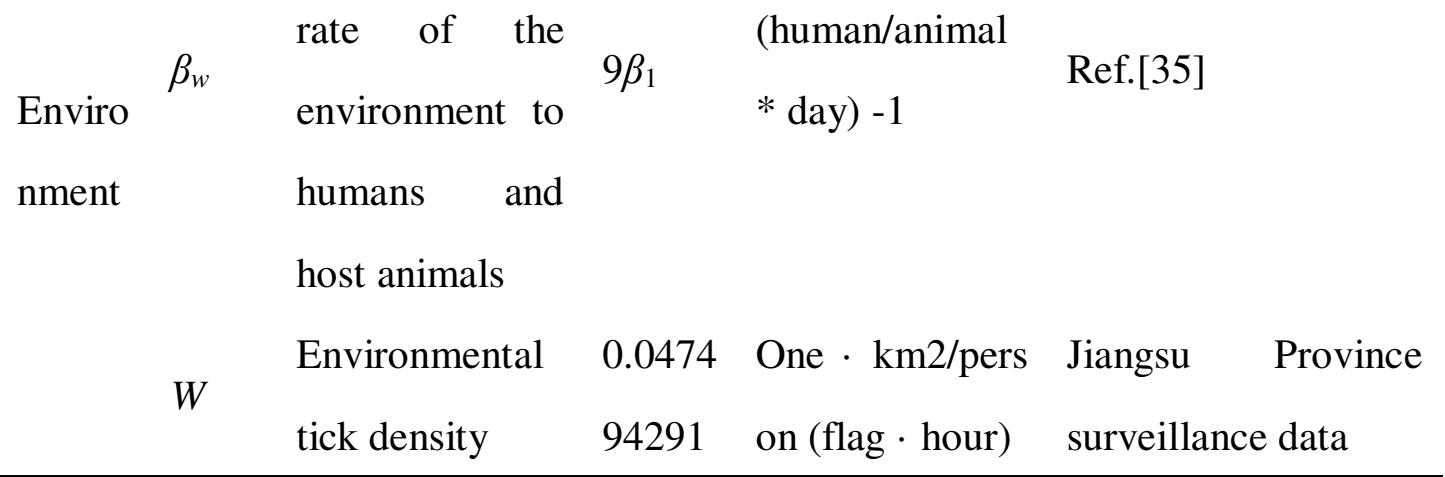


659 Table 2 Combined transmissibility matrix

\begin{tabular}{llllllll}
\hline & $\beta_{1}$ & $\beta_{3}$ & $\beta_{w}$ & $\beta_{21}$ & $\beta_{31}$ & $\beta_{32}$ & $\beta_{23}$ \\
\hline$\beta_{1}$ & 1 & $2 \beta_{1}$ & $9 \beta_{1}$ & $8 \beta_{1}$ & $2 \beta_{1}$ & $8 \beta_{1}$ & $8 \beta_{1}$ \\
$\beta_{3}$ & $1 / 2 \beta_{3}$ & 1 & $9 / 2 \beta_{3}$ & $4 \beta_{3}$ & $1 \beta_{3}$ & $4 \beta_{3}$ & $4 \beta_{3}$ \\
$\beta_{w}$ & $1 / 9 \beta_{w}$ & $2 / 9 \beta_{w}$ & 1 & $8 / 9 \beta_{w}$ & $2 / 9 \beta_{w}$ & $8 / 9 \beta_{w}$ & $8 / 9 \beta_{w}$ \\
$\beta_{21}$ & $1 / 8 \beta_{21}$ & $1 / 4 \beta_{21}$ & $9 / 8 \beta_{21}$ & 1 & $1 / 4 \beta_{21}$ & $1 \beta_{21}$ & $1 \beta_{21}$ \\
$\beta_{31}$ & $1 / 2 \beta_{31}$ & $1 \beta_{31}$ & $9 / 2 \beta_{31}$ & $4 \beta_{31}$ & 1 & $4 \beta_{31}$ & $4 \beta_{31}$ \\
$\beta_{32}$ & $1 / 8 \beta_{32}$ & $1 / 4 \beta_{32}$ & $9 / 8 \beta_{32}$ & $1 \beta_{32}$ & $1 / 4 \beta_{32}$ & 1 & $1 \beta_{32}$ \\
$\beta_{23}$ & $1 / 8 \beta_{23}$ & $1 / 4 \beta_{23}$ & $9 / 8 \beta_{23}$ & $1 \beta_{23}$ & $1 / 4 \beta_{23}$ & $1 \beta_{23}$ & 1 \\
\hline
\end{tabular}

660

661 
662 Table 3 Model fitting effects 2011-2019

\begin{tabular}{ccc}
\hline Year & $R^{2}$ & $P$ \\
\hline 2011 & 0.573878 & $<0.05$ \\
2012 & 0.29952 & $<0.05$ \\
2013 & 0.482155 & $<0.05$ \\
2014 & 0.764983 & $<0.05$ \\
2015 & 0.408163 & $<0.05$ \\
2016 & 0.767604 & $<0.05$ \\
2017 & 0.776788 & $<0.05$ \\
2018 & 0.801787 & $<0.05$ \\
2019 & 0.758567 & $<0.05$ \\
\hline
\end{tabular}

663

664 
665 Table 4 Interventional effect of cutting off different transmission routes on SFTS

\begin{tabular}{|c|c|c|c|c|}
\hline $\begin{array}{l}\text { Transmission } \\
\text { route }\end{array}$ & $\begin{array}{l}\text { Cumulative number of } \\
\text { cases }\end{array}$ & $\begin{array}{l}\text { Total attack } \\
\text { rate }\end{array}$ & $\begin{array}{l}\text { Peak } \\
\text { time }\end{array}$ & $\begin{array}{l}\text { Peak attack } \\
\text { rate }\end{array}$ \\
\hline$\beta_{1}$ & 0 & 0 & 0 & 0 \\
\hline$\beta_{3}$ & 671 & 0 & 205 & 0 \\
\hline$\beta_{w}$ & 0 & 0 & 198 & 0 \\
\hline$\beta_{21}$ & 671 & 0 & 205 & 0 \\
\hline$\beta_{31}$ & 671 & 0 & 205 & 0 \\
\hline$\beta_{23}$ & 671 & 0 & 205 & 0 \\
\hline$\beta_{32}$ & 671 & 0 & 205 & 0 \\
\hline$\beta_{3} \beta_{w}$ & 0 & 0 & 198 & 0 \\
\hline$\beta_{3} \beta_{21}$ & 671 & 0 & 205 & 0 \\
\hline$\beta_{3} \beta_{31}$ & 671 & 0 & 205 & 0 \\
\hline$\beta_{3} \beta_{23}$ & 671 & 0 & 205 & 0 \\
\hline$\beta_{3} \beta_{32}$ & 671 & 0 & 205 & 0 \\
\hline$\beta_{w} \beta_{21}$ & 0 & 0 & 198 & 0 \\
\hline$\beta_{w} \beta_{31}$ & 0 & 0 & 10 & 0 \\
\hline$\beta_{w} \beta_{23}$ & 0 & 0 & 198 & 0 \\
\hline$\beta_{w} \beta_{32}$ & 0 & 0 & 198 & 0 \\
\hline$\beta_{21} \beta_{23}$ & 671 & 0 & 205 & 0 \\
\hline$\beta_{21} \beta_{31}$ & 671 & 0 & 205 & 0 \\
\hline$\beta_{21} \beta_{32}$ & 671 & 0 & 205 & 0 \\
\hline$\beta_{31} \beta_{23}$ & 671 & 0 & 205 & 0 \\
\hline$\beta_{31} \beta_{32}$ & 671 & 0 & 205 & 0 \\
\hline$\beta_{23} \beta_{32}$ & 671 & 0 & 205 & 0 \\
\hline$\beta_{3} \beta_{w} \beta_{21}$ & 0 & 0 & 198 & 0 \\
\hline$\beta_{3} \beta_{w} \beta_{31}$ & 0 & 0 & 10 & 0 \\
\hline$\beta_{3} \beta_{w} \beta_{23}$ & 0 & 0 & 198 & 0 \\
\hline$\beta_{3} \beta_{w} \beta_{32}$ & 0 & 0 & 198 & 0 \\
\hline
\end{tabular}




\begin{tabular}{|c|c|c|c|c|}
\hline$\beta_{3} \beta_{21} \beta_{31}$ & 671 & 0 & 205 & 0 \\
\hline$\beta_{3} \beta_{21} \beta_{32}$ & 671 & 0 & 205 & 0 \\
\hline$\beta_{3} \beta_{21} \beta_{23}$ & 671 & 0 & 205 & 0 \\
\hline$\beta_{3} \beta_{31} \beta_{23}$ & 671 & 0 & 205 & 0 \\
\hline$\beta_{3} \beta_{31} \beta_{32}$ & 671 & 0 & 205 & 0 \\
\hline$\beta_{3} \beta_{23} \beta_{32}$ & 671 & 0 & 205 & 0 \\
\hline$\beta_{w} \beta_{21} \beta_{31}$ & 0 & 0 & 10 & 0 \\
\hline$\beta_{w} \beta_{21} \beta_{23}$ & 0 & 0 & 198 & 0 \\
\hline$\beta_{w} \beta_{31} \beta_{23}$ & 0 & 0 & 10 & 0 \\
\hline$\beta_{w} \beta_{21} \beta_{32}$ & 0 & 0 & 198 & 0 \\
\hline$\beta_{w} \beta_{31} \beta_{32}$ & 0 & 0 & 10 & 0 \\
\hline$\beta_{w} \beta_{23} \beta_{32}$ & 0 & 0 & 198 & 0 \\
\hline$\beta_{21} \beta_{31} \beta_{23}$ & 671 & 0 & 205 & 0 \\
\hline$\beta_{21} \beta_{31} \beta_{32}$ & 671 & 0 & 205 & 0 \\
\hline$\beta_{21} \beta_{23} \beta_{32}$ & 671 & 0 & 205 & 0 \\
\hline$\beta_{31} \beta_{23} \beta_{32}$ & 671 & 0 & 205 & 0 \\
\hline$\beta_{3} \beta_{w} \beta_{21} \beta_{31}$ & 0 & 0 & 7 & 0 \\
\hline$\beta_{3} \beta_{w} \beta_{21} \beta_{23}$ & 0 & 0 & 198 & 0 \\
\hline$\beta_{3} \beta_{w} \beta_{21} \beta_{32}$ & 0 & 0 & 198 & 0 \\
\hline$\beta_{3} \beta_{w} \beta_{23} \beta_{32}$ & 0 & 0 & 198 & 0 \\
\hline$\beta_{3} \beta_{w} \beta_{31} \beta_{23}$ & 0 & 0 & 10 & 0 \\
\hline$\beta_{3} \beta_{w} \beta_{31} \beta_{32}$ & 0 & 0 & 10 & 0 \\
\hline$\beta_{3} \beta_{21} \beta_{31} \beta_{23}$ & 671 & 0 & 205 & 0 \\
\hline$\beta_{3} \beta_{21} \beta_{23} \beta_{32}$ & 671 & 0 & 205 & 0 \\
\hline$\beta_{3} \beta_{21} \beta_{31} \beta_{32}$ & 671 & 0 & 205 & 0 \\
\hline$\beta_{3} \beta_{31} \beta_{23} \beta_{32}$ & 671 & 0 & 205 & 0 \\
\hline$\beta_{w} \beta_{21} \beta_{31} \beta_{23}$ & 0 & 0 & 10 & 0 \\
\hline$\beta_{w} \beta_{21} \beta_{31} \beta_{32}$ & 0 & 0 & 10 & 0 \\
\hline$\beta_{w} \beta_{21} \beta_{23} \beta_{32}$ & 0 & 0 & 198 & 0 \\
\hline
\end{tabular}




\begin{tabular}{lllll}
$\beta_{w} \beta_{31} \beta_{23} \beta_{32}$ & 0 & 0 & 10 & 0 \\
$\beta_{21} \beta_{31} \beta_{23} \beta_{32}$ & 671 & 0 & 205 & 0 \\
\cline { 2 - 5 }$\beta_{3} \beta_{w} \beta_{21} \beta_{31} \beta_{23}$ & 0 & 0 & 10 & 0 \\
$\beta_{3} \beta_{w} \beta_{21} \beta_{31} \beta_{32}$ & 0 & 0 & 10 & 0 \\
$\beta_{3} \beta_{w} \beta_{21} \beta_{23} \beta_{32}$ & 0 & 0 & 198 & 0 \\
$\beta_{3} \beta_{w} \beta_{31} \beta_{23} \beta_{32}$ & 0 & 0 & 10 & 0 \\
$\beta_{3} \beta_{21} \beta_{31} \beta_{23} \beta_{32}$ & 671 & 0 & 205 & 0 \\
$\beta_{w} \beta_{21} \beta_{31} \beta_{23} \beta_{32}$ & 0 & 0 & 10 & 0 \\
$\beta_{3} \beta_{w} \beta_{21} \beta_{31} \beta_{23} \beta_{32}$ & 0 & 0 & 10 & 0 \\
\hline
\end{tabular}

666 


\begin{tabular}{|c|c|c|c|c|}
\hline Intervention & $\begin{array}{l}\text { Cumulative number } \\
\text { of cases }\end{array}$ & $\begin{array}{l}\text { Total } \\
\text { attack } \\
\text { rate }\end{array}$ & Peak time & $\begin{array}{l}\text { Peak attack } \\
\text { rate }\end{array}$ \\
\hline$\gamma=1 / 14 \phi=0$ & 530 & 0.00 & 197 & 0 \\
\hline$\gamma=1 / 14 \phi=0.5 \%$ & 531 & 0.00 & 197 & 0 \\
\hline$\gamma=1 / 14 \phi=5 \%$ & 530 & 0.00 & 197 & 0 \\
\hline$\gamma=1 / 14 \phi=50 \%$ & 530 & 0.00 & 197 & 0 \\
\hline$\gamma=1 / 12 \phi=0$ & 531 & 0.00 & 197 & 0 \\
\hline$\gamma=1 / 12 \phi=0.5 \%$ & 531 & 0.00 & 197 & 0 \\
\hline$\gamma=1 / 12 \phi=5 \%$ & 531 & 0.00 & 197 & 0 \\
\hline$\gamma=1 / 12 \phi=50 \%$ & 531 & 0.00 & 197 & 0 \\
\hline$\gamma=1 / 10 \phi=0$ & 531 & 0.00 & 197 & 0 \\
\hline$\gamma=1 / 10 \phi=0.5 \%$ & 531 & 0.00 & 197 & 0 \\
\hline$\gamma=1 / 10 \phi=5 \%$ & 531 & 0.00 & 197 & 0 \\
\hline$\gamma=1 / 10 \phi=50 \%$ & 531 & 0.00 & 197 & 0 \\
\hline$\gamma=1 / 8 \phi=0$ & 531 & 0.00 & 197 & 0 \\
\hline$\gamma=1 / 8 \phi=0.5 \%$ & 531 & 0.00 & 197 & 0 \\
\hline$\gamma=1 / 8 \phi=5 \%$ & 531 & 0.00 & 197 & 0 \\
\hline$\gamma=1 / 8 \phi=50 \%$ & 531 & 0.00 & 197 & 0 \\
\hline$\gamma=1 / 6 \phi=0$ & 531 & 0.00 & 197 & 0 \\
\hline$\gamma=1 / 6 \phi=0.5 \%$ & 531 & 0.00 & 197 & 0 \\
\hline$\gamma=1 / 6 \phi=5 \%$ & 531 & 0.00 & 197 & 0 \\
\hline$\gamma=1 / 6 \phi=50 \%$ & 531 & 0.00 & 197 & 0 \\
\hline$\gamma=1 / 4 \phi=0$ & 531 & 0.00 & 197 & 0 \\
\hline$\gamma=1 / 4 \phi=0.5 \%$ & 531 & 0.00 & 197 & 0 \\
\hline$\gamma=1 / 4 \phi=5 \%$ & 531 & 0.00 & 197 & 0 \\
\hline$\gamma=1 / 4 \phi=50 \%$ & 531 & 0.00 & 197 & 0 \\
\hline
\end{tabular}


670 Table 6 Interventional effect of taking different $r$

\begin{tabular}{lllll}
\hline $\begin{array}{l}\text { Isolation } \\
\text { ratio }\end{array}$ & $\begin{array}{l}\text { Cumulative number of } \\
\text { cases }\end{array}$ & $\begin{array}{l}\text { Cumulative attack } \\
\text { rate }\end{array}$ & $\begin{array}{l}\text { Peak } \\
\text { time }\end{array}$ & $\begin{array}{l}\text { Peak attack } \\
\text { rate }\end{array}$ \\
\hline $50 \%$ & 530 & 0.00 & 201 & 0.00 \\
$60 \%$ & 530 & 0.00 & 201 & 0.00 \\
$70 \%$ & 530 & 0.00 & 201 & 0.00 \\
$80 \%$ & 530 & 0.00 & 201 & 0.00 \\
$90 \%$ & 530 & 0.00 & 201 & 0.00 \\
$98 \%$ & 530 & 0.00 & 201 & 0.00 \\
\hline
\end{tabular}

671

672 


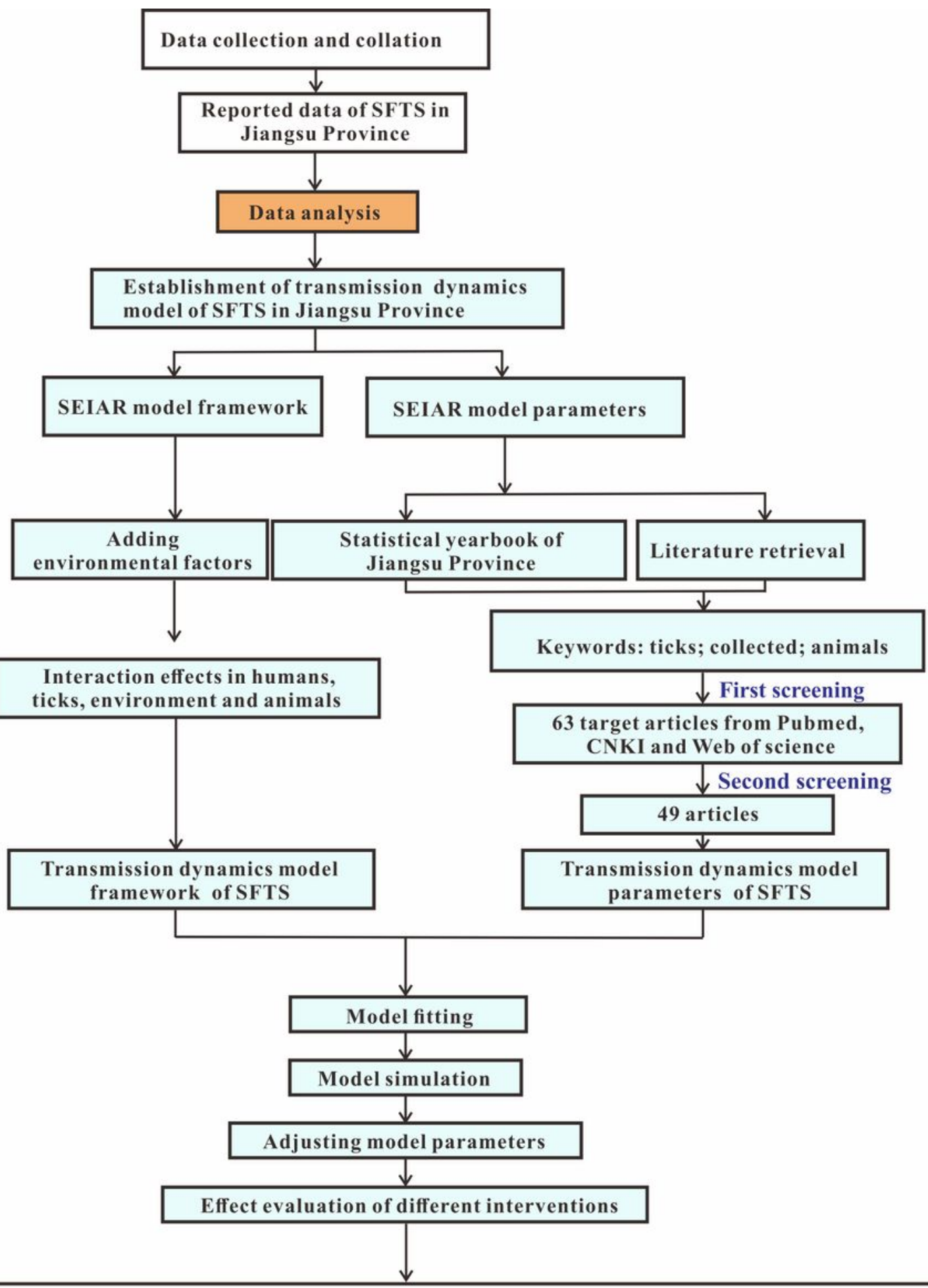

Transmission dynamics model of Severe Fever with Thrombocytopenia Syndrome in Jiangsu province

\section{Figure 1}




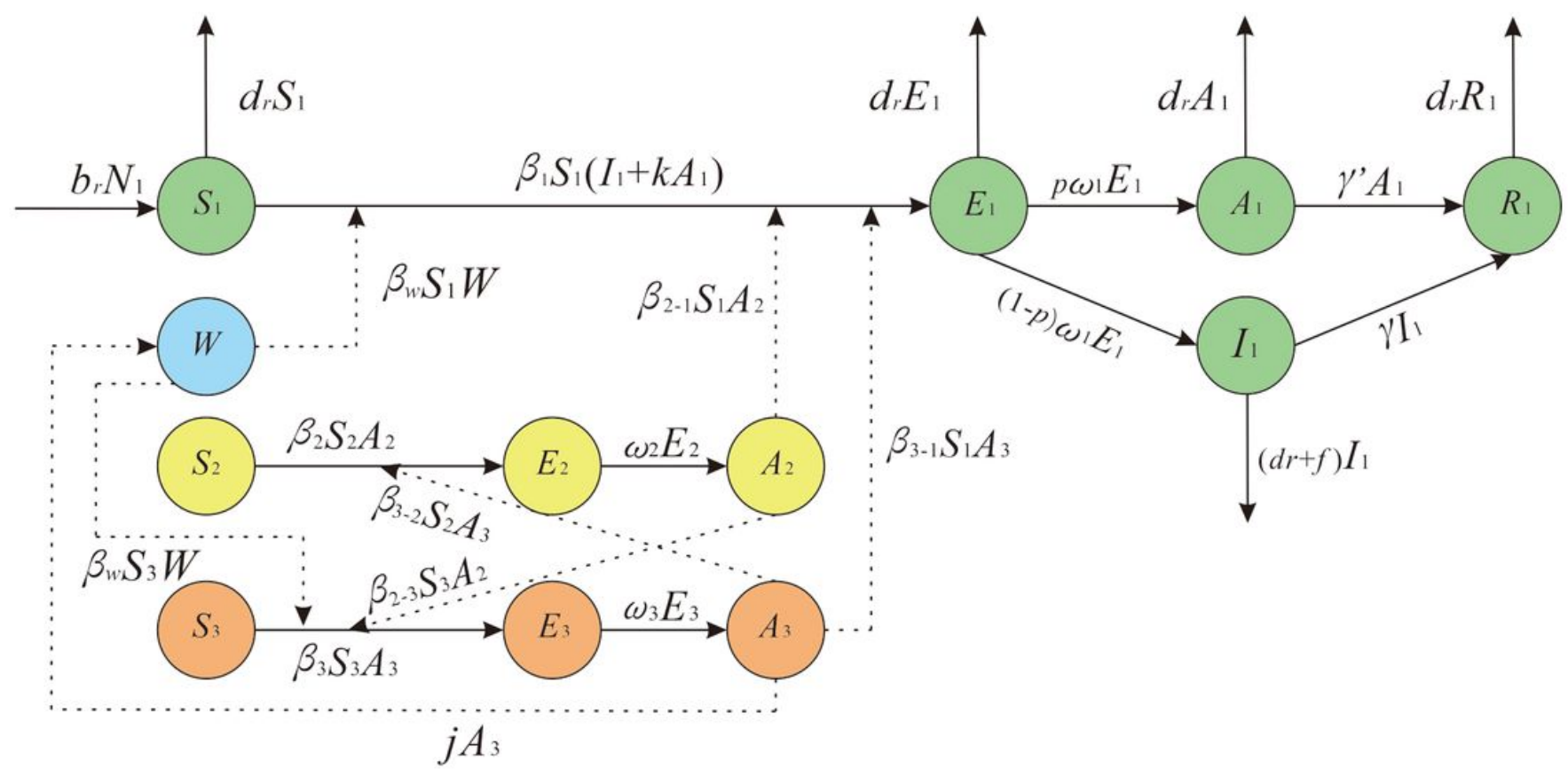

\section{Figure 2}

SFTS without intervention model

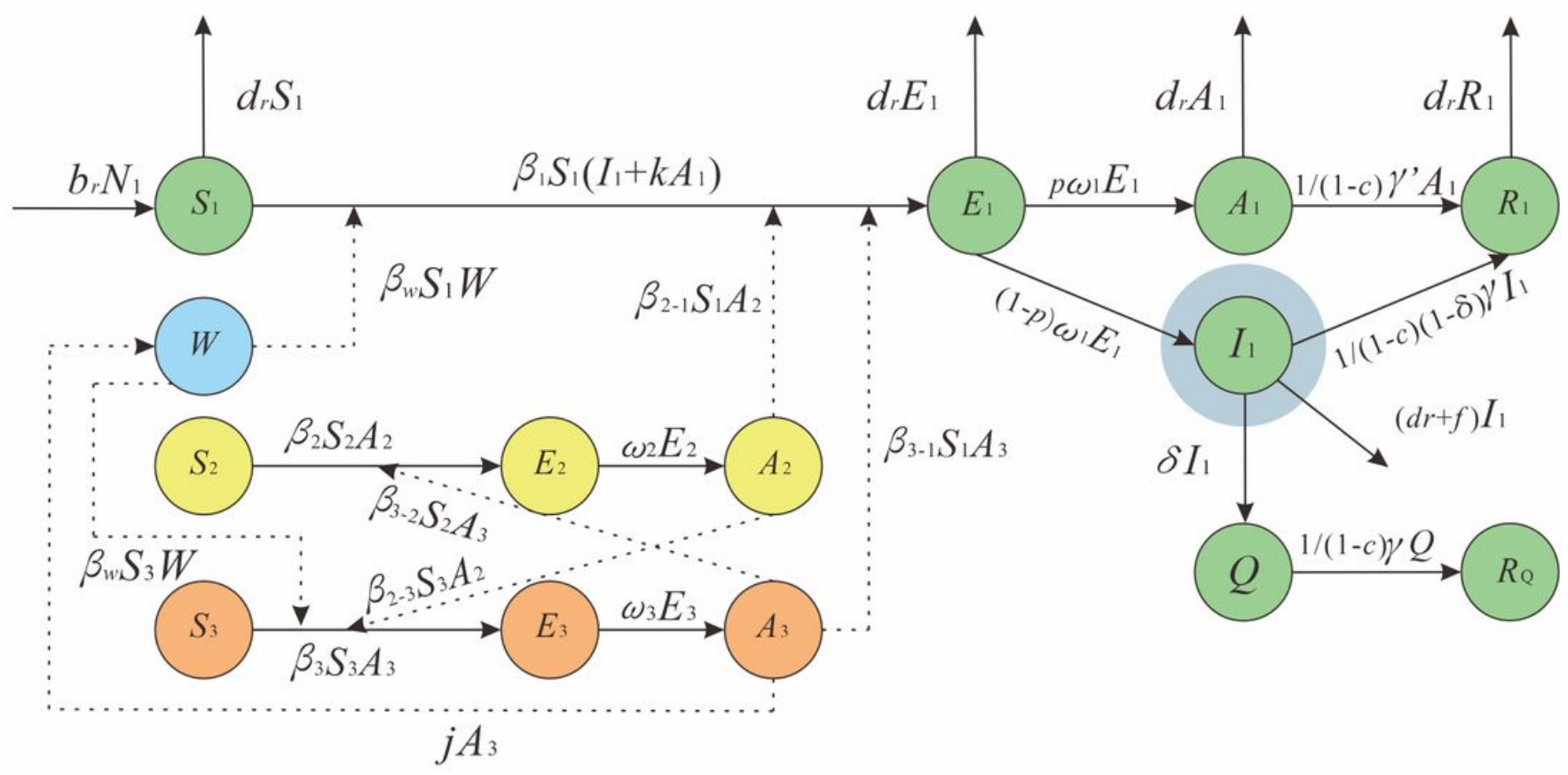

Figure 3

SFTS with intervention model 


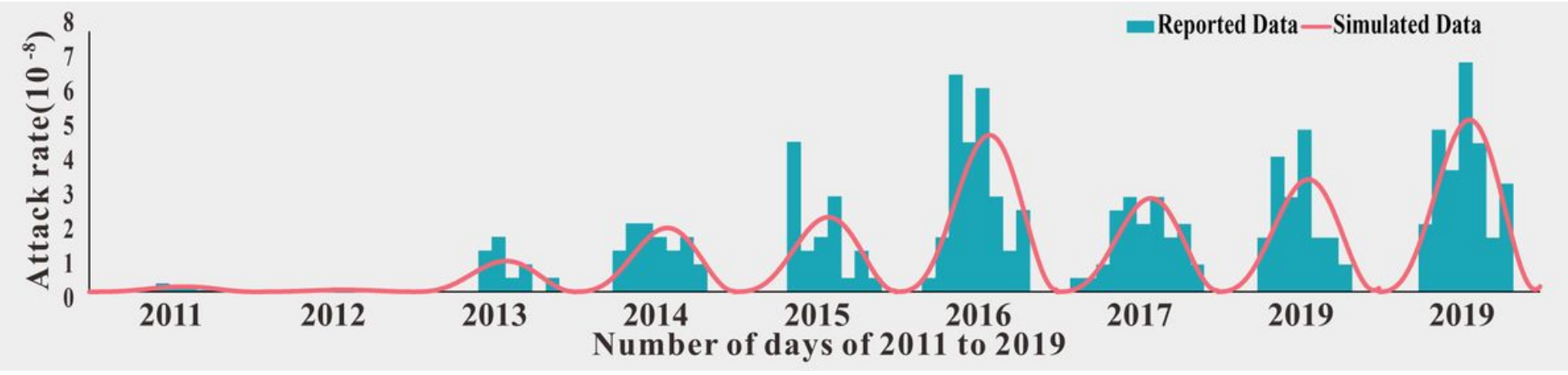

Figure 4

Model fitting result of incidence of 2011-2019 in Jiangsu Province

\section{Supplementary Files}

This is a list of supplementary files associated with this preprint. Click to download.

- GraphicalAbstract.jpg 